\title{
Pharyngeal pouch (Zenker's diverticulum)
}

\author{
M A Siddiq, S Sood, D Strachan
}

\begin{abstract}
Pharyngeal pouches occur most commonly in elderly patients (over 70 years) and typical symptoms include dysphagia, regurgitation, chronic cough, aspiration, and weight loss. The aetiology remains unknown but theories centre upon a structural or physiological abnormality of the cricopharyngeus. A diagnosis is easily established on barium studies. Treatment is surgical via an endoscopic or external cervical approach and should include a cricopharyngeal myotomy. Unfortunately pharyngeal pouch surgery has long been associated with significant morbidity, partly due to the surgery itself and also to the fact that the majority of patients are elderly and often have general medical problems. External approaches are associated with higher complication rates than endoscopic procedures. Recently, treatment by endoscopic stapling diverticulotomy has becoming increasingly popular as it has distinct advantages, although long term results are not yet available. The small risk of developing carcinoma within a pouch that is not excised remains a contentious issue and is an argument for long term follow up or treating the condition by external excision, particularly in younger patients. (Postgrad Med f 2001;77:506-511)
\end{abstract}

Keywords: Zenker's diverticulum; surgical procedures: endoscopic; surgical stapling

Historical perspective

The first pharyngeal pouch was described by Ludlow in $1769,{ }^{1}$ who reported an abnormal dilatation of the posterior pharyngeal wall in a postmortem examination of a patient who had complained of dysphagia during life. The pharyngeal pouch was reclassified by and has since been eponymously linked with Zenker after his and Von Ziemssens concise pathological description of 34 patients with a protrusion of pharyngeal mucosa on the dorsal wall, immediately proximal to the transition from hypopharynx into oesophagus. ${ }^{2}$ The site of herniation through Killian's dehiscence, between the thyropharyngeal and cricopharyngeal fibres of the inferior constrictor, was reported in 1908 (fig $1) .^{3}$

However it was not until 1886 that Wheeler performed the first successful excision. ${ }^{4}$ Several surgical procedures have since been described for the treatment of pharyngeal pouches. These include diverticulectomy, ${ }^{56}$ diverticulopexy, ${ }^{78}$ diverticular inversion, ${ }^{9}{ }^{10}$ cricopharyngeal myotomy, ${ }^{11}$ and endoscopic diverticulotomy. ${ }^{12}{ }^{13}$ Mosher (1917) is widely credited as being the first to use an endoscopic technique to treat pharyngeal pouches by dividing the common septum between the oesophagus and pouch. ${ }^{14}$ In his publication Mosher reported good results on four patients and in view of these results he continued with this method until the seventh patient unfortunately developed mediastinitis and died. Dohlman redescribed and modified the endoscopic technique and reported on 39 patients on whom he had used this procedure since $1935 .{ }^{15}$ He used a specially designed double lipped hypopharyngoscope inserting the upper lip into the oesophagus and the shorter lower lip into the diverticulum, coagulated the common septum with insulated forceps, and divided it using a diathermy knife and electrocautery instruments. By 1960 this series had risen to 100 cases with a recurrence rate of $7 \%$ and no associated deaths or serious complications. ${ }^{16}$ The endoscopic method has been further modified to include the use of an operating microscope, ${ }^{17}$ carbon dioxide, ${ }^{17-19}$ and potassium titanyl phosphate (KTP) ${ }^{20}$ lasers to divide the muscular septum. More recently in 1993, endoscopic stapling diverticulotomy using a linear transecting and stapling device has been introduced by Martin-Hirsch and Newbegin ${ }^{12}$ in the UK and by Collard et $a l^{13}$ in Belgium.

\section{Aetiology}

A clear understanding of the pathogenesis of pharyngeal pouches is lacking. Several theories exist on the formation of these pulsion diverticulae and centre upon the structural or physiological abnormality of the cricopharyngeus muscle. The upper oesophageal sphincter between the hypopharynx and oesophagus is usually closed by resting muscle tone and opened during swallowing by relaxation of muscle fibres together with cephalic displacement of the larynx creating a negative pressure. Wouters and Van Overbeek proposed that an anatomical predisposition to a large Killian's

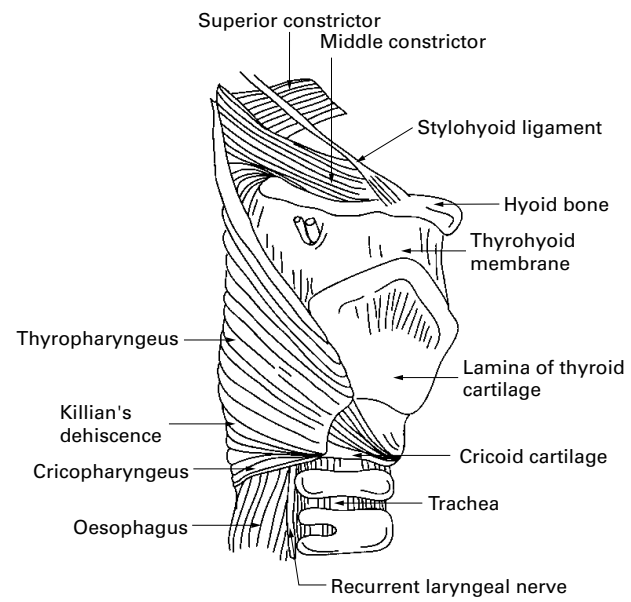

Figure 1 Lateral view of pharynx showing Killian's dehiscence. 
dehiscence, which lies between the propulsive oblique fibres of the thyropharyngeus and the horizontal fibres of the cricopharyngeus, which have a sphincteric action, plays a prominent part in mucosal herniation. ${ }^{21}$ They added that this is further supported by their finding of a familial occurrence of these diverticulae. It has been recently postulated that the pathophysiology of pharyngeal pouches involves altered compliance of the cricopharyngeus muscle detected as impaired sphincter opening or raised intrabolus pressure using specialised cricopharyngeal manometric recordings. ${ }^{22}$ Shaw et al supported the finding of poor upper oesophageal sphincter compliance and added that hypopharyngeal intrabolus pressure is a useful indicator of upper sphincter compliance which normalises after surgery. ${ }^{23}$ Cook et al using videoradiography and manometry found intrabolus pressures were greater in patients with a diverticulum compared with an age matched healthy population and concluded that a disorder of diminished upper oesophageal opening was the cause. ${ }^{24}$ Histologically it has been observed that in patients with a pharyngeal pouch, the cricopharyngeus demonstrates fibroadipose tissue replacement and fibre degeneration which it is proposed may account for the observed diminished upper oesophageal sphincter opening. ${ }^{25}$

The interrelationship between gastrooesophageal reflux and/or hiatus hernia remains unresolved. ${ }^{26}$ However an association has been reported between them ${ }^{27}$ and indeed in one study ${ }^{28}$ the incidence of hiatus hernia was more than double in patients with diverticulae compared with a control group.

\section{Pathology}

Pharyngeal diverticulae may be posterior, posterolateral, or lateral (pharyngocoele) but the most commonly encountered type is the posterior pulsion diverticulum. ${ }^{29}$ There is usually a single opening at Killian's dehiscence, although the presence of a double pharyngeal pouch has been reported. ${ }^{30}$ The majority of pharyngeal pouches protrude to the left side and it has been suggested that the handedness of the patient may determine the side on which the pouch occurs. ${ }^{31}$

The histology of the pouch when excised usually shows a sac containing an epithelial lining that is stratified squamous epithelium, and the submucosa often shows fibrous tissue surrounding it. ${ }^{29}$ Near to the neck of the sac scanty muscle fibres may be found in the wall. Rarely a squamous cell carcinoma or a carcinoma in situ may be present in the pouch.

Other histological varieties are extremely rare-a case of an ulcerated pouch with submucosal infiltration by plasma cells, lymphocytes, and eosinophils has been reported. ${ }^{29}$

\section{Clinical features}

The disorder occurs more commonly in the northern part of Europe than the southern part: it is common in the United States, Canada, and Australia but rare in Japan and Indonesia. ${ }^{32} \mathrm{~A}$ community study from the UK estimates an annual incidence of two per
Box 1: Typical signs and symptoms of pharyngeal pouches

- Age (over 70 years).

- Dysphagia.

- Regurgitation.

- Aspiration.

- Cough.

- Borborygmi.

- Choking.

- Halitosis.

- Weight loss.

- Hoarseness.

100000 people per year. ${ }^{33}$ However the true incidence of the condition may be difficult to ascertain as there may be a significant number of elderly patients with pouches who may not seek medical advice as they can have minimal symptoms. Pharyngeal pouches are uncommon before the age of 40 , usually occurring in the seventh decade or later and being more common in males than females. ${ }^{34}$

Typical symptoms include dysphagia (a consistent symptom), regurgitation of undigested food, choking, borborygmi in the cervical region, chronic cough, chronic aspiration due to overspill of contents from the pouch, halitosis, weight loss, and less commonly hoarseness (box 1). As the pouch enlarges symptoms generally become more severe and large diverticulae can result in malnutrition. ${ }^{29}$ The duration of symptoms at presentation may vary from weeks to several years. ${ }^{29}$ Examination findings are few and may include emaciation and rarely a swelling maybe felt in the neck which may gurgle on palpation (Boyce's sign).

A possible malignancy in a pouch should be suggested when there has been a sudden increase in the severity of symptoms, particularly progressive dysphagia or aphagia or if there is pain, haemoptysis, or more marked regurgitation of food. ${ }^{35}$

Pharyngeal pouches are readily diagnosed on barium studies, which should delineate the pouch well. The radiological study is incomplete if it does not include the lower oesophagus, stomach and duodenum, in order to look for any other abnormalities such as hiatus hernia or reflux oesophagitis. Contrast videofluoroscopy allows constant monitoring of the swallowing mechanism which is valuable as single shot barium swallows may miss a small diverticulum. Barium swallow studies may demonstrate a filling defect which does not move between films (as a food bolus does) and any loss of the smooth contour of the interior of the pouch should raise the suspicion of a carcinoma. ${ }^{35}$ If present it is most commonly noted in the distal two thirds of the pouch but can be easily missed.

There have been attempts to classify the size of pouch as demonstrated by contrast radiology. Morton and Bartley describe small pouches as less than $2 \mathrm{~cm}$, medium pouches as $2-4 \mathrm{~cm}$, and large pouches as greater than 


\author{
Box 2: Surgical treatment methods \\ Endoscopic \\ - Dohlman's (electrocoagulation). \\ - Dohlman's (laser). \\ - Stapling. \\ External \\ - Cricopharyngeal myotomy. \\ - Diverticulectomy. \\ - Inversion. \\ - Diverticulopexy (suspension).
}

$4 \mathrm{~cm} .{ }^{9}$ Van Overbeek and Groote classify the pouch as small if it is less than one cervical vertebrae in size and large if it is greater than three vertebrae. ${ }^{32}$

\section{Treatment}

The treatment of choice for an established pharyngeal pouch is surgical and the approach maybe external or endoscopic (box 2). Obviously each case should be judged on its own merit with the patient aware of the potential risks and benefits of an operation. However in some situations such as in an elderly, medically unfit patient with minimal symptoms, no treatment except careful observation maybe indicated.

As most surgeons consider an abnormality of the cricopharyngeus muscle to be the main factor in the formation of pharyngeal pouches, surgical treatments usually include a cricopharyngeal myotomy. Unfortunately pharyngeal pouch surgery has long been associated with a risk of significant complications. This is partly due to the type of surgery involved and partly to the frailty of the population operated upon. In the 1996/7 report from the National Confidential Enquiry into Perioperative Deaths on head and neck surgery the advisors were surprised by the mortality rates of $1 \%-2 \%$, the majority of which were after open surgery. ${ }^{36}$ The report concluded that subspecialisation within otolaryngology departments would seem appropriate and that endoscopic surgery is a quicker, less invasive, and safe technique. Many surgical treatments have been developed for treatment of the condition but there is as yet no consensus as to the best option.

Both external and endoscopic procedures are associated with risks and complications. External procedures involve an approach to the pouch via a lateral cervical approach, identifying the pouch and performing a cricopharyngeal myotomy. The pouch can itself then be treated by inversion, mobilisation, and suspension or excised and closed by a suturing technique or a stapling technique. In some cases, such as small pouches, an external cricopharyngeal myotomy on its own may be deemed adequate. The patient is usually fed via a nasogastric tube for five to seven days after which oral feeding is resumed.

Endoscopic procedures involve division of the common muscular and mucosal septum that lies between the oesophagus and pouchessentially performing an internal cricopharyngeal myotomy and creating a single lumen.
Although the pouch has not been removed, it no longer fills and food passes into the oesophageal lumen with relief of symptoms. Patients can usually resume oral intake within 24 hours.

Dohlman's technique divides the septum using electrocautery. ${ }^{16}$ This technique was widely used until Van Overbeek in $1984^{17}$ introduced microendoscopic surgery using the operating microscope and either electrocautery or a carbon dioxide laser to achieve division of the septum. His experience with 274 patients confirmed microendoscopic treatment as a reliable treatment method. He felt that using a microscope was a significant improvement in endoscopic surgery and felt little difference in patients who had undergone carbon dioxide laser or diathermy treatment except that pain was probably less in patients undergoing laser in the first few postoperative days. His recent published experience has now extended to 545 cases. ${ }^{37}$ A modification of this method described by Kuhn and Bent employed a $\mathrm{KTP} / 532$ laser and they reported no major complications on a small series of 10 patients. ${ }^{20}$

Recently endoscopic stapling devices have become increasingly popular. A distending Stortz double lipped hypopharyngoscope is used to visualise the common septum. It allows passage of the stapling gun and is based on the original Dohlman's hypopharyngoscope. An autosuture disposable surgical staple is passed through the endoscope with one jaw in the pouch and one into the oesophagus thus isolating the common septum. The gun is fired thus cutting the septum and simultaneously sealing the edges with staples. ${ }^{12}$ This achieves opening of the neck of the pouch and division of the cricopharyngeus with closure of the party wall between the pouch and oesophagus thereby sealing the potential opening into the mediastinum. The advantages of using a stapling device over laser or diathermy are a reduced risk of perforation and subsequent mediastinitis as the divided edges of the septum are sealed by the staples, better haemostasis and avoidance of thermal damage to the recurrent laryngeal nerve. ${ }^{38}$ In addition, endoscopic stapling has the advantages of a short anaesthetic time (which is particularly important in the elderly or medically unfit), early resumption of oral intake, short inpatient stay, minimal postoperative pain and straightforward revision surgery, ${ }^{13}{ }^{38-41}$ which in the case of excision can be difficult as scar tissue makes identification of the diverticulum hazardous (see table 1). However, there are limitations with the endoscopic approach as exposing both the diverticulum or oesophagus may prove difficult due to patient anatomy or underlying disease such as kyphosis. ${ }^{39}$ Also in patients with a small pouch there can be difficulty in fitting the stapling gun around the common septum, which may result in an insufficient cricopharyngeal myotomy. In addition, there is no specimen available for pathological assessment, although the pouch should be carefully inspected before stapling with biopsies taken of any suspicious areas of mucosa. Despite these limitations this technique has been recommended as the treatment 
Table 1 Advantages/disadvantages of endoscopic v external surgery

\begin{tabular}{ll}
\hline External & Endoscopic \\
\hline Longer procedure & $\begin{array}{l}\text { Short procedure and anaesthetic time } \\
\text { Longer hospital stay (typically 5-7 days) with nasogastric feeds for } 5 \text { days }\end{array}$ \\
$\begin{array}{l}\text { Higher complication rate } \\
\text { Specimen available for histological assessment to exclude carcinoma }\end{array}$ & $\begin{array}{l}\text { Lower complication rate } \\
\text { No histological assessment of pouch }\end{array}$ \\
Proved long term satisfactory results & Long term results of stapling awaited, although good results reported with laser \\
Revision surgery can be difficult & Revision surgery straightforward
\end{tabular}

Box 3: Complications of pharyngeal pouch surgery

- Recurrent laryngeal nerve damage.

- Pouch perforation.

- Mediastinitis.

- Pharyngeal fistula.

- Wound infection.

- Pharyngeal stenosis.

- Recurrence of pouch.

of choice for all pouches by several authors..$^{38-41}$ The amount of septum that can safely be divided is a matter of judgment for the surgeon and is probably related to the experience of the surgeon. ${ }^{42}$ The reported complication rates are very low but long term outcomes are yet to be reported..$^{384} 41$

Diverticulectomy has the advantage that it completely removes the pouch and technically can be used to manage all sizes of pouch, although this maybe difficult in the smallest pouches. A diverticulectomy also eliminates any theoretical risk of carcinoma which may exist in residual mucosa of a pouch that may remain after endoscopic treatment. ${ }^{35}$ However it does carry a higher complication rate including wound infections, haematoma, fistula formation, surgical emphysema, mediastinitis, and vocal cord paralysis (box 3). $.^{33} 43-45$ It also requires a longer anaesthetic time, making it less appropriate for the elderly or frail patient and a longer hospital stay.

Inversion of a pouch is usually used to treat small to medium sized pouches. Bowdler and Stell reported a reduced mortality, complication rate, and hospital stay with inversion surgery over diverticulectomy. ${ }^{46}$ They proposed that inversion should be the treatment of choice. However they excluded patients who underwent endoscopic procedures. A more recent report concurred with this conclusion but also stated that inversion is better if pouches are not too large and not too longstanding to risk leaving subclinical carcinoma. ${ }^{9}$ In a recent review of current practice in pharyngeal pouch surgery among otolaryngologists, general surgeons and cardiothoracic surgeons, ${ }^{47}$ excision was found to be the commonest procedure performed by general surgeons but the Dohlman's procedure was popular among otolaryngologists probably because of their familiarity with rigid endoscopy. It was also mentioned that endoscopic stapling diverticulotomy was becoming increasingly popular.

Both external excision and endoscopic methods can be used to treat recurrences of the condition. However, endoscopic methods have been proposed as a safer alternative. Koay et al have reported improved symptoms after restapling in recurrent diverticulae without increased morbidity. ${ }^{42}$

In comparing the treatment modalities, most series show that the endoscopic and external approaches are equally effective treatments. ${ }^{33}{ }^{37}{ }^{48-50}$ However there is no doubt that the external approach has a higher complication rate, including vocal cord paralysis, mediastinitis, fistula formation, glottic oedema, and stricture formation. $.^{33} 43-45$ However these studies were retrospective and lacked criteria for selecting patients and the procedures were performed by a large number of surgeons from several specialties. It is thus difficult to draw firm conclusions as to which is the better method of treatment, although in centres performing endoscopic diverticulotomy as a first line treatment for pharyngeal pouch, the results seem to be consistently good..$^{37}{ }^{41}{ }^{51}$ However as endoscopic stapling is a relatively new technique, complications may not as yet be well reported.

There is a definite learning curve with endoscopic procedures and therefore higher success rates can be expected from larger series of patients. This is borne out by Van Overbeek who achieved a low complication rate in one of the largest published series of 545 patients. ${ }^{37}$

Assessment of treatment outcome can be made clinically. There is no role for postoperative contrast studies as they bear little correlation to symptoms and lax mucosa that remains following stapling may appear to be a residual pouch in an asymptomatic patient. ${ }^{52}$ Therefore the need for further treatment should be guided clinically by patient symptoms.

Patient satisfaction studies have been used to evaluate treatment methods. Wouters and Van Overbeek reported a $99 \%$ satisfaction rate after endoscopic diathermy or laser myotomy performed on 507 patients. ${ }^{53}$ In this series there was an $8 \%$ incidence of significant complications, a $2 \%$ rate of mediastinitis and one death. In a comparison of excision and endoscopic stapling, Van Eeden et al reported improved symptoms in $88 \%$ of endoscopically treated patients and $70 \%$ of those undergoing excision with a 5\% complication rate for endoscopic procedures and a $23 \%$ rate for external procedures. ${ }^{40}$ Short and long term follow ups of patients testify to a satisfactory outcome in $90 \%$ or more patients treated by endoscopic or external surgery, ${ }^{33}$ although there are at present no long term results of endoscopic stapling diverticulotomy. 


\section{Questions (answers on p 511)}

1. What is the site of herniation of a pharyngeal pouch?

(A) Cricopharyngeus.

(B) Thyroid cartilage.

(C) Middle constrictor.

(D) Between the cricopharyngeus and thyropharyngeus fibres of the inferior constrictor muscle.

(E) Upper oesophagus.

2. What are the possible aetiological factors in the development of a pharyngeal pouch?

(A) Age (over 50 years).

(B) Female sex.

(C) Poor upper oesophageal sphincter pressures.

(D) Hiatus herniation and oesophageal reflux.

(E) Large Killian's dehisence.

3. What are the common symptoms that a pharyngeal pouch may present with?

(A) Dysphagia.

(B) Voice change.

(C) Odynophagia.

(D) Regurgitation.

(E) Weight loss.

4. What symptoms or signs may raise the possibility of a malignancy within a pouch?

(A) Aphagia or rapidly progressing dysphagia.

(B) Pain.

(C) Haemoptysis.

(D) Smooth contour on videofluoroscopic examination.

(E) Dyspnoea.

5. Treatment of a pharyngeal pouch, if indicated, is surgical. What are the possible options?

(A) Pouch excision.

(B) Pouch inversion.

(C) Pharyngo-oesophagectomy.

(D) Endoscopic stapling

(E) Endoscopic electrocautery (Dohlman's procedure).

6. Early studies have suggested a reduced complication rate with the endoscopic approach to treatment, but what are the other potential benefits of this approach?

(A) Shorter anaesthetic time.

(B) Reduced recurrence rate.

(C) Early resumption of oral intake.

(D) Various surgical specialties can perform it.

(E) Shorter hospital stay.

7. Which of the following are the disadvantages of the endoscopic approach?

(A) Due to neck anatomy (for example, kyphosis) access may be too limited to carry out the procedure.

(B) Difficult to treat large pouches.

(C) Increased risk of mediastinitis.

(D) Increased risk of damage to the recurrent laryngeal nerve.

(E) No specimen is sent for pathological assessment.
Carcinoma and carcinoma in situ are both rare. The main predisposing factor in carcinoma developing within pharyngeal pouches is thought to be chronic inflammation of the pouch lining over many years, secondary to food retention. ${ }^{35}$ To date 45 cases of carcinoma have been reported in the English language literature. ${ }^{54-57}$ The diagnosis is often made at surgery when cleaning of the pouch and careful examination with an oesophagoscope or Hopkins rod should be performed prior to any definitive surgical procedure. However cases of carcinoma in situ or small carcinomas may not be detected radiologically or by endoscopic examination. In a recent review by Bradley et al, two cases of carcinoma in situ were reported. ${ }^{35}$ Both of these had not been suspected clinically or found on endoscopy and the diagnosis was established by histopathological examination. Thus such lesions can be potentially left in pouches treated endoscopically. A further potential problem in patients treated by endoscopic surgery is that if the pouch persists despite a lack of symptoms, will it still become irritated by food bolus and are they therefore still at risk of a carcinoma? In such cases it could be argued that patients should be informed of the risk of carcinoma development in the pouch years later if it is not excised at the first presentation. ${ }^{35}$ It is for this reason that some authors propose that patients less than 65 years should undergo excision of the pouch with a long cricopharyngeal myotomy and pathological examination of the pouch. ${ }^{35}$ Long term follow up should be considered in these cases and endoscopic examination of the whole oesophagus should be undertaken if symptoms persist or recur. ${ }^{35}$ Such long term follow up of endoscopic cases will hopefully clarify the true risk of carcinoma developing in such instances.

\section{Conclusion}

The surgical procedures used to treat pharyngeal pouches vary widely. The preferred treatment depends on individual experience and proponents of all methods report good results. Endoscopic stapling diverticulotomy is becoming increasingly popular and is the treatment of choice in many centres, although the potential risk of a subclinical carcinoma being missed in a pouch which is then not excised remains. Hence long term follow up studies which are available for all other treatment methods are necessary for endoscopic stapling as these may change our future management approach to the pharyngeal pouch.

\footnotetext{
1 Ludlow A. A case of obstructed deglutition from a preternatural dilatation of and bag formed in the pharynx. Medical observations and enquiries by a society of physicians in London. 2nd Ed. 1769;3:85-101.

2 Zenker FA, von Ziemssen H. Krankheiten des Oesophagus. In: von Ziemssen H, ed. Handbuch der Speciellen Pathologie and Therapie. Vol 7 (suppl). Leipzig: FCW Vogel, 1877: $1-87$.

3 Killian G. Ueber den mund der speiserohre. Z Ohrenheilkd, Krankheiten Luftwege 1908;55:1-41.

4 Wheeler WI. Pharyngocoele and dilation of the pharynx, with existing diverticulum at lower portion of pharynx lying posterior to the oesophagus, cured by pharyngotomy, being posterior to the oesophagus, cured by pharyngotomy, being
the first of the kind recorded. Dublin fournal of Medical Science $1886 ; 82: 349-56$.

5 Todd GB. The treatment of pharyngeal pouch. 7 Laryngol Otol 1974;88:307-15.
} 
6 Mackay IS. The treatment of pharyngeal pouch. F Laryngol Otol 1976;90:183-90.

7 Laccourreye O, Menard M, Cauchois R, et al. Esophageal diverticulum: diverticulopexy versus diverticulectomy. Laryngoscope 1994;104:889-92.

8 Konowitz PM, Biller HF. Diverticulopexy and cricopharyngeal myotomy: treatment for the high-risk patient with a pharyngoesophageal (Zenker's) diver

Head Neck Surg 1993;100:146-53.
9 Morton RP, Bartley JR. Inversion of Zenker's diverticula: the preferred option. Head Neck 1993;15:253-6.

10 Johnson JT, Weissman J. Diverticular imbrication and myotomy for Zenker's. Laryngoscope 1992;102:1377-8.

11 Schmit PJ, Zuckerbraun L. Treatment of Zenker's diverticula by cricopharyngeus myotomy under local anaesthesia. Am Surg 1992;58:710-16.

12 Martin-Hirsch DP, Newbegin CJR. Autosuture GIA gun: a new application in the treatment of hypopharyngeal diverticula. J Laryngol Otol 1993;107:723-5.

13 Collard JM, Otte JB, Kestens PJ. Endoscopic stapling technique of esophagodiverticulostomy for Zenker's diverticulum. Ann Thorac Surg 1993;56:573-6.

14 Mosher HP. Webs and pouches of the esophagus: their diagnosis and treatment. Surg Gynecol Obstet 1917;25:175-87.

15 Dohlman G. Endoscopic operations for hypopharyngeal diverticula. Proceedings of the Fourth International Congress on Otolaryngology. London, 1948: 715-17.

16 Dohlman G, Mattsson O. The endoscopic operation for hypopharyngeal diverticula. $\mathcal{F}$ Laryngol Otol 1960;71:74452.

17 van Overbeek JJM, Hoeksema PE, Edens ET. Microendoscopic surgery of the hypopharyngeal diverticulum using electrocoagulation or carbon dioxide laser. Ann Otol Rhinol Laryngol 1984;93:34-6.

18 Holinger LD, Benjamin B. New endoscope for (laser) endoscopic diverticulotomy. Ann Otol Rhinol Laryngol 1987;96: 658-60.

19 Benjamin B, Gallagher R. Microendoscopic laser diverticulotomy for hypopharyngeal diverticulum. Ann Otol Rhinol ulotomy for hypopharynge
Laryngol 1993;102:675-9.

20 Kuhn FA, Bent JP 3rd. Zenker's diverticulotomy using the KTP/532 laser. Laryngoscope 1992;102:946-50.

21 Wouters B, Van Overbeek JJM. Pathogenesis and endoscopic treatment of the hypopharyngeal (Zenker's) diverscopic treatment of the hypopharyngeal (Zen

22 Peters JH, Mason R. The physiopathological basis for Zenker's diverticulum. Chirurg 1999;70:741-6.

23 Shaw DW, Cook IJ, Jamieson GG, et al. Influence of surgery on deglutitive upper oesophageal sphincter mechanics in Zenker's diverticulum. Gut 1996;38:806-11.

24 Cook IJ, Gabb M, Panagopoulos V, et al. Pharyngeal (Zenker's diverticulum) is a disorder of upper esophageal sphincter opening. Gastroenterology 1992;103:1229-35.

25 Cook IJ, Blumbergs P, Cash K, et al. Structural abnormalities of the cricopharyngeus muscle in patients with pharyn-
geal (Zenker's) diverticulum. $\mathcal{F}$ Gastroenterol Hepatol 1992;7: 556-62.

26 Feussner H, Siewert JR. Zenker's diverticulum and reflux. Hepatogastroenterology 1992;39:100-4.

27 Resouly A, Braat J, Jackson A, et al. Pharyngeal pouch: link Resouly A, Braat J, Jackson A, et al. Pharyngeal pouch: link
with reflux and oesophageal dysmotility. Clin Otolaryngol with reflux and

28 Gage-White L. Incidence of Zenker's diverticulum with hiatus hernia. Laryngoscope 1988;98:527-30.

29 Bowdler DA. Pharyngeal pouches. In Kerr A, ed. Scott Brown's textbook of otorhinolaryngology. 6th Ed. Oxford: Butterworth-Heinmann, 1997: 1-21.

30 Meehan T, Henein RR. An unusual pharyngeal pouch. $\mathcal{F}$ Laryngol Otol 1992;106:1002-3.

31 Stafford ND, Moore-Gillon V, McKelvie P. Handedness and the side on which pharyngeal pouches occur. BMF 1984;288:815-16.

32 van Overbeek JJM, Groote AD. Zenker's diverticulum. Current Opinion in Otolaryngology, Head and Neck Surgery 1994; 2:55-8.

33 Laing MR, Murthy P, Ah-See KW, et al. Surgery for pharyngeal pouch: audit of management with short and pharyngeal pouch: audit of management with short an

34 Maran AGD, Wilson JA, Al Muhanna AH. Pharyngeal diverticula. Clin Otolaryngol 1986;11:219-25.
35 Bradley PJ, Kochaar A, Quraishi MS. Pharyngeal pouch carcinoma: real or imaginary risks? Ann Otol Rhinol Laryngol 108:1027-32.

36 Resouly A. Pharyngeal pouch surgery. The report of the National Confidential Enquiry into Perioperative Deaths. London: Royal College of Surgeons of England, 1996/7, 32.

37 Van Overbeek JJM. Meditation on the pathogenesis of hypopharyngeal (Zenker's) diverticulum and a report of endoscopic treatment in 545 patients. Ann Otol Rhinol Laryngol 1994;103:178-85.

38 Koay CB, Bates GJ. Endoscopic stapling diverticulotomy for pharyngeal pouch. Clin Otolaryngol 1996;21:371-6.

39 Scher RL, Richtsmeier WJ. Long-term experience with endoscopic-staple assisted esophagodiverticulostomy for Zenker's diverticulum. Laryngoscope 1998;108:200-5.

40 van Eeden S, Lloyd RV, Tranter RM. Comparison of the endoscopic stapling technique with more established procedures for pharyngeal pouches: results and patient satisfaction survey. F Laryngol Otol 1999;113:237-40.

41 Baldwin DL, Toma AG. Endoscopic stapled diverticulotomy: a real advance in the treatment of hypopharyngeal diverticulum. Clin Otolaryngol 1998;23:

42 Koay CB, Commins D, Bates GJ. The role of endoscopic stapling diverticulotomy in recurrent pharyngeal pouch. $\mathcal{F}$ Laryngol Otol 1998;112:954-5.

43 Zbaren P, Schar P, Tschopp L, et al. Surgical treatment of Zenker's diverticulum: transcutaneous diverticulectomy versus microendoscopic myotomy of the cricopharyngeal muscle with CO2 laser. Otolaryngol Head Neck Surg 1999;121:482-7.

44 Feeley MA, Righi PD, Weisberger EC, et al. Zenker's diverticulum: analysis of surgical complications from diverticulectomy and cricopharyngeal myotomy. Laryngoscope 1999;109:858-61.

45 Aggerholm K, Illum P. Surgical treatment of Zenker's diverticulum. F Larynol Otol 1990;104:312-14.

46 Bowdler DA, Stell PM. Surgical management of posterior pulsion pharyngeal diverticula: inversion versus one-stage pulsion pharyngeal diverticula: inversi

47 Koay CB, Sharp HR, Bates GJ. Current practice in pharyngeal pouch surgery in England and Wales. Ann R Coll Surg Engl 1997;79:190-4.

48 Lippert BM, Folz BJ, Gottschlich S, et al. Microendoscopic treatment of the hypopharyngeal diverticulum with the CO2 laser. Lasers Surg Med 1997;20:394-01.

49 Bonafede JP, Lavertu P, Wood BG, et al. Surgical outcome in 87 patients with Zenker's diverticulum. Laryngoscope 1997; 107:720-5.

50 Payne WS. The treatment of pharyngoesophageal diverticulum: the simple and complex. Hepatogastroenterology 1992;39:109-14.

51 Wayman DM, Byl FM, Adour KK. Endoscopic diverticulotomy for the treatment of Zenker's diverticulum. Otolayngol Head Neck Surg 1991;104:448-52.

52 Ong CC, Elton PG, Mitchell D. Pharyngeal pouch endoscopic stapling-are post-operative barium swallow radiographs of any value?

53 Wouters B, Van Overbeek JJM. Endoscopic treatment of the hypopharyngeal (Zenker's) diverticulum. Hepatogastroenterology 1992;39:105-8

54 Bowdler DA, Stell PM. Carcinoma arising in posterior pharyngeal pulsion diverticulum (Zenker's diverticulum). $\mathrm{Br} f$ Surg 1987;74:561-3.

55 Stevens DJ. Carcinoma of the pharyngeal pouch. F Laryngol Otol 1989;103:695-7.

56 Burton MJ, Lund WS. Pharyngeal pouch carcinoma: two unusual cases. 7 Laryngol Otol 1990;104:821-3.

57 Jorgensen T, Martinez Ramos C, Nunez Pena JR, et al. Carcinoma in a long-standing Zenker's diverticulum. Rev Esp Enferm Dig 1994;85:203-7.

Answers $(T=$ true, $F=$ false $)$

1. (A) F, (B) F, (C) F, (D) T, (E) F; 2. (A) F, (B) F, (C) T, (D) $\mathrm{T}$, (E) T. 3. (A) T, (B) F, (C) F, (D) T, (E) T; 4. (A) T, (B) T, T, (B) F, (C) T, (D) F, (E) T; 7. (A) T, (B) F, (C) F, (D) F, (E) $\mathrm{T}$ 